\title{
Constraints on the variations of fundamental couplings by stellar models
}

\author{
A. Coc ${ }^{1}$, S. Ekström ${ }^{2}$, P. Descouvemont ${ }^{3}$ and E. Vangioni ${ }^{4}$ \\ ${ }^{1}$ Centre de Spectrométrie Nucléaire et de Spectrométrie de Masse (CSNSM), UMR 8609, \\ CNRS/IN2P3 and Université Paris Sud 11, Bâtiment 104, 91405 Orsay Campus, France \\ ${ }^{2}$ Geneva Observatory, University of Geneva, Maillettes 51, 1290 Sauverny, Switzerland \\ ${ }^{3}$ Physique Nucléaire Théorique et Physique Mathématique, C.P. 229, Université Libre de \\ Bruxelles (ULB), B-1050 Brussels, Belgium \\ ${ }^{4}$ Institut dAstrophysique de Paris, UMR-7095 du CNRS, Université Pierre et Marie Curie, 98 \\ bis bd Arago, 75014 Paris, France
}

\begin{abstract}
The effect of variations of the fundamental constants on the thermonuclear rate of the triple alpha reaction, ${ }^{4} \mathrm{He}(\alpha \alpha, \gamma){ }^{12} \mathrm{C}$, that bridges the gap between ${ }^{4} \mathrm{He}$ and ${ }^{12} \mathrm{C}$ is investigated. We follow the evolution of 15 and $60 M_{\odot}$ zero metallicity star models, up to the end of core helium burning. They are assumed to be representative of the first, Population III stars undergoing a very peculiar evolution due to the absence of initial CNO elements (zero metallicity). The calculated oxygen and carbon abundances resulting from helium burning can then be used to constrain the variations of the fundamental constants.
\end{abstract}

Keywords. cosmology: theory, Pop III stars, nuclear reactions, nucleosynthesis, abundances

The equivalence principle is a cornerstone of metric theories of gravitation and in particular of General Relativity. It follows that by testing the constancy of fundamental constants one actually performs a test of General Relativity, that can be extended on astrophysical and cosmological scales. We consider here (see details in Coc et al. 2009, and Ekström et al. 2009), the very first generation of stars which are thought to have been formed a few $10^{8}$ years after the big bang, at a redshift of $z \sim 10-15$, and with zero initial metallicity. The synthesis of complex elements in stars (mainly the possibility of the $3 \alpha$-reaction at the origin of the procuction of ${ }^{12} \mathrm{C}$ ) sets constraints on the values of the fine structure and strong coupling constants. This reaction is indeed very sensitive to the position of a resonance corresponding to the "Hoyle state" in ${ }^{12} \mathrm{C}$. In order to analyze its variation with the nuclear interaction, we have used a nuclear microscopic cluster model and performed model calculations of a 15 and $60 M_{\odot}$ zero metallicity star until to the end of core helium burning. We deduce the limits on the variation of the nuclear interaction to insure that the $\mathrm{C} / \mathrm{O}$ ratio be of the order of unity. Depending on models, this can be relateded to limits on the variations of the fine structure constant.

\section{References}

Coc, A., Ekström, S., Descouvemont, P., Meynet, G., Olive, K., Uzan, J.-Ph., \& Vangioni, E., proceedings of JD 9 IAU Symposium, Rio 2009, to appear in Mem. S.A. It..

Ekström, S., Coc, A., Descouvemont, P., Meynet, G., Olive, K., Uzan, J.-Ph., \& Vangioni, E., 2009 in preparation. 